\title{
Tumores Phyllodes de mama: clínica, tratamiento y pronóstico. Hospital Universitario de Canarias, España
}

\author{
Olivia Orribo M. ${ }^{1}$, Raysé Rodríguez ${ }^{1}{ }^{1}$, Leticia Melgar $V^{2}{ }^{2}$ Francisco Fernández . $^{1}$, \\ Francisco Javier De La Torre F. \\ ${ }^{1}$ Servicio de Ginecología y Obstetricia, ${ }^{2}$ Servicio de Anatomía Patológica, Hospital Universitario de Canarias, España.
}

\section{RESUMEN}

El tumor Phyllodes de mama es un tumor poco frecuente que se engloba dentro de los tumores fibroepiteliales de mama, con un amplio abanico de presentación clínica y comportamiento biológico. Se clasifican en benignos, borderline o malignos, en base a factores histológicos. Las formas menos agresivas o benignas presentan un comportamiento similar al de los fibroadenomas, sin embargo las formas malignas suelen presentar un comportamiento más agresivo. El pronóstico de este tipo de tumor es favorable, con una recurrencia local del $15 \%$ de forma global y recurrencia a distancia entre 5-10\%. El tratamiento se basa en la escisión quirúrgica de la lesión, acompañada de radioterapia e incluso quimioterapia en las formas más agresivas. Presentamos la revisión de 13 casos clínicos de esta entidad diagnósticados en nuestro centro entre 2001-2009.

\section{PALABRAS CLAVE: Tumor Phyllodes, cáncer de mama, tumores fibroepiteliales, mamografía, biopsia}

\section{SUMMARY}

Phyllodes tumor of the breast is a rare tumor, which is part of fibroepithelial breast tumors with a wide range of clinical presentation and biological behavior. They are classified into benign, borderline or malignant based on histologic factors. The less aggressive or benign forms show a more behavior to that of fibroadenomas, but malignant forms usually show a more aggressive behavior. The prognosis of this tumor is favorable, with local recurrence in $15 \%$ overall and distant recurrence between $5-10 \%$ globally. The treatment relies on surgical excision of the lesion accompanied by radiotherapy and chemotherapy even in the most aggressive. We present a review of 13 cases of this entity diagnosed in our center between 2001-2009.

KEY WORD: Phyllodes tumor, breast cancer, fibroepithelial tumor, mammography, biopsy

\section{INTRODUCCIÓN}

El tumor Phyllodes es una neoplasia muy rara dentro de los tumores de mama. Fue descrita por primera vez por Johannes Müller en 1838 (1), denominándolo inicialmente cistosarcoma phyllodes, término que se dejó de utilizar ya que realmente no son auténticos sarcomas dado su comportamiento biológico y su origen celular.

Se trata de un tumor bifásico que presenta elementos estromales y epiteliales. Afecta principalmente a mujeres en torno a la cuarta década de 
la vida. El pronóstico de los tumores phyllodes, en general, es favorable aunque varía en función de si se trata de formas benignas, borderline o malignas, con una tasa de supervivencia libre de enfermedad a los 5 años de hasta el $80 \%$ para las formas malignas (2). El tratamiento debe de ser conservador dado su comportamiento biológico y su baja agresividad en formas benignas y borderline.

El objetivo de esta comunicación es presentar una serie de 13 casos de tumores Phyllodes de mama diagnosticados en nuestro centro entre los años 2001 y 2009.

\section{PACIENTES Y MÉTODO}

Se realiza estudio retrospectivo de 13 tumores Phyllodes de mama diagnosticados en nuestro medio entre el año 2001-2009. Se evalúan características demográficas, tamaño de la lesión, diagnóstico mamográfico, resultado del estudio anatomopatológico, tipo de tratamiento y sobrevida a 2 años. El análisis de las variables se realizó a través del programa SPSS $12.0 \circledast$.

\section{RESULTADOS}

La edad media al diagnóstico de las pacientes fue de 42,2 años (rango: 30-61 años), observando mayor edad en pacientes con formas más agresivas con respecto a las pacientes con formas benignas (45 años en formas malignas vs 42 años en las benignas). El $93 \%$ las pacientes fueron remitidas a nuestras consultas por presentar nódulo en mama. Las pacientes refirieron como única sintomatología bulto mamario de rápido crecimiento en 12 de los 13 casos. El tamaño medio de la lesión a la exploración fue de $3,8 \mathrm{~cm}$ (rango: 1,5-7 cm). En el $82 \%$ de los casos el nódulo se localizaba en cuadrantes superoexternos. No se hallaron diferencias significativas en cuanto a la mama afecta.

En 10 casos se realizó mamografía, clasificadas en $2 / 3$ de los casos como BIRADS III y en $1 / 3$ restante como BIRADS IV (encontramos que en uno de los casos clasificado como BIRADS IV se confirmó posteriormente como tumor Phyllodes maligno). En los 3 casos en los que no se realizó mamografía se realizó ecografía debido a la edad de la paciente (< de 35 años). Como otras pruebas complementarias en el $54 \%$ de los casos realizamos punción-aspiración con aguja fina (PAAF), en un $18 \%$ biopsia con aguja gruesa (BAG) y en un $9 \%$ punción-aspiración con aguja fina mediante guía ecográfica (ECO-PAAF). Dentro de los resultados citológicos y de anatomía patológica llama la atención, que en el $50 \%$ las pruebas complementarias previas a la extirpación de la lesión informan de lesión compatible con tumor phyllodes, en un $20 \%$ el resultado no era concluyente y en el 30\% restante fibroadenoma. Inicialmente en todos los casos se procedió a realizar tumorectomía de la lesión.

En nuestra serie nos encontramos que en 3 de los casos se trataban de formas malignas por lo que tras llevar los casos a Cómite Oncológico se decidió ampliación quirúrgica (mastectomía). En ningún caso se realizó determinación de ganglio centinela, debido a que por lo general no suele existir afectación ganglionar. En las formas malignas en todos los casos las pacientes recibieron tratamiento complementario con radioterapia y quimioterapia. La tasa de supervivencia global a los 2 años del diagnóstico fue del $100 \%$ para las formas benignas frente a un $66,6 \%$ para las formas malignas (Tabla I).

\section{DISCUSIÓN}

El tumor Phyllodes de mama representa menos del $0,5 \%$ de todos los tumores de mama $(3,4)$. Se engloban dentro de los tumores mixtos de mama. El término Phyllodes se debe a las proyecciones papilares, características que se observan al microscopio.

Este tipo de tumores se da en mujeres en torno a los 40 años, con un rango de edad que varía entre los 10-82 años $(5,6)$, tal y como se comprueba en nuestra serie. Revisiones recientes apuntan que a mayor edad en el momento del diagnóstico se relaciona con un mayor grado histológico (7), como se comprueba en nuestra revisión.

Debido a su baja incidencia no existen muchos estudios en cuanto a su etiología y factores pronósticos, sin embargo actualmente solo se ha identificado como factor predisponente el Síndrome de Li- Fraumeni (8), síndrome autosómico dominante, en el que existe una sobreexpresión del P53 que favorece la aparición de múltiples tumores entre los cuáles se ve una predisposición a desarrollar tumores Phyllodes de mama. Se he descrito también como factor predisponente antecedentes de fibroadenoma.

Clínicamente, se suele manifestar como un nódulo firme, liso, multinodular, bien delimitado, móvil y por lo general no doloroso; con un tamaño variable en torno a los $4-7 \mathrm{~cm}$ de media (6), como se comprueba en nuestra revisión. Se caracteriza por tener un rápido crecimiento que provoca adelgazamiento en la piel dando lugar al característico retículo venoso. Aunque a la exploración hasta en un $20 \%$ de los casos se pueden palpar adenopatías, generalmente secundario a un proceso infeccioso, la afectación ganglionar es rara (3). 


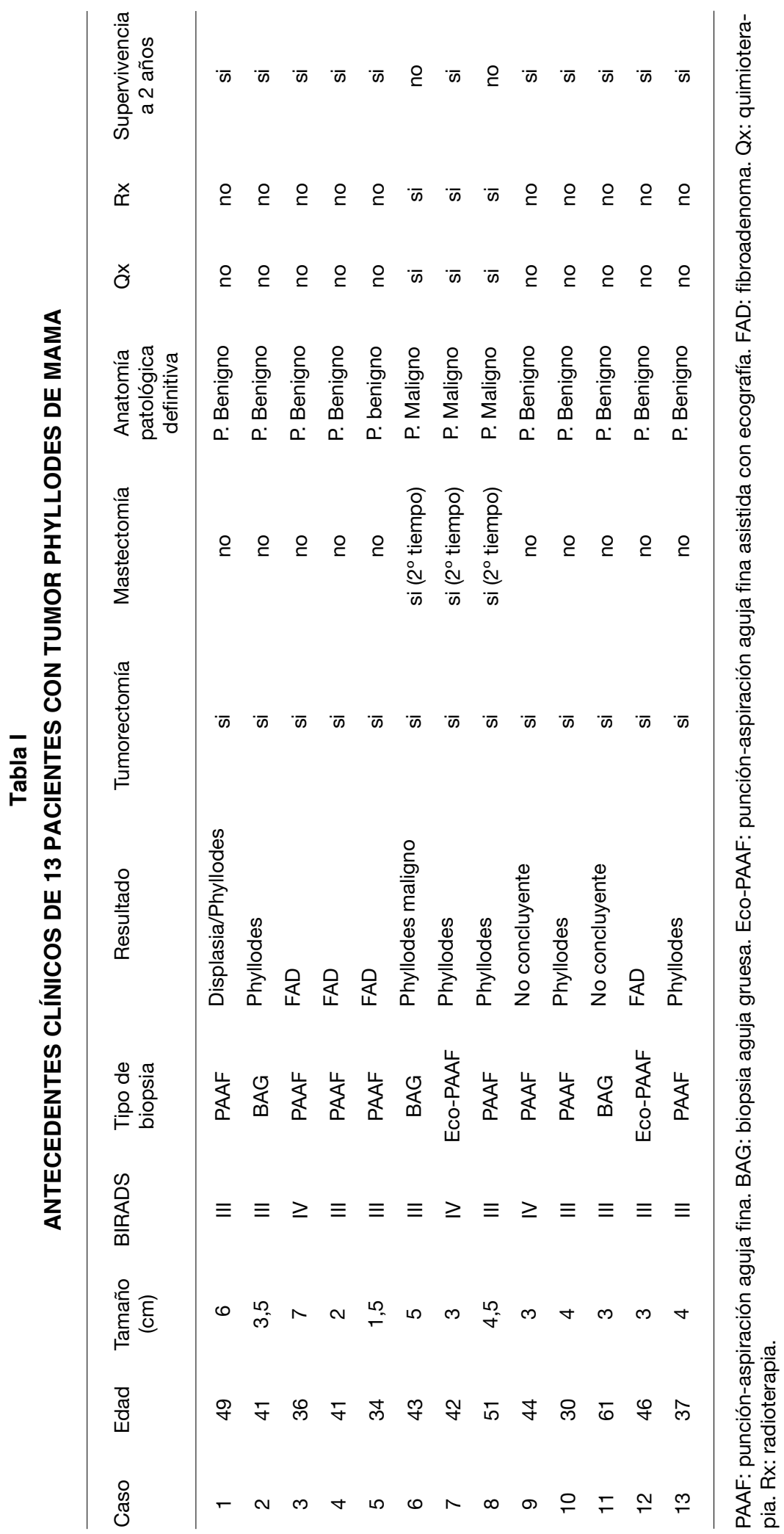


Hay que tener en cuenta que en 2 de cada 10 casos se trata de tumores no palpables (2), de ahí la importancia de complementar la exploración con pruebas de imagen, mamografía (Figura 1) y ecografía (Figura 2), sin embargo es importante recalcar que éstas no tienen hallazgos patognomónicos, siendo imposible en algunas ocasiones diferenciar mediante dichas técnicas un fibroadenoma de un tumor phyllodes.

El diagnóstico de confirmación es anatomopatológico (9), de ahí la importancia de realizar BAG, PAAF o Eco-PAAF en todo nódulo de mama. Se ha visto que existe un $30 \%$ de falsos negativos al realizar una biopsia de mama, por lo que ante todo nódulo sólido de rápido crecimiento o sintomático se recomienda repetir la biopsia a pesar de tener una previa negativa $(10,11)$.

Macroscópicamente se parece a un fibroadenoma, por lo general de mayor tamaño, de aspecto polilobulado que recuerda a una coliflor con zonas de necrosis y hemorragia (Figura 3).

Microscópicamente se caracteriza por presentar proyecciones papilares de epitelio estromal intercalando zonas de hiperplasia y atipia. El componente estromal es el que nos va a permitir diferencia los fibroadenomas de los tumores Phyllodes, y dentro de éstos las formas benignas de las malignas (5). Histológicamente se clasifican en formas benignas (representan alrededor del 50\%), borderline y malignas en función $(12,13)$ : atipia del estroma celular, actividad mitótica y grado de infiltración, y presencia o ausencia de sobrecrecimiento estromal (Figura 4).

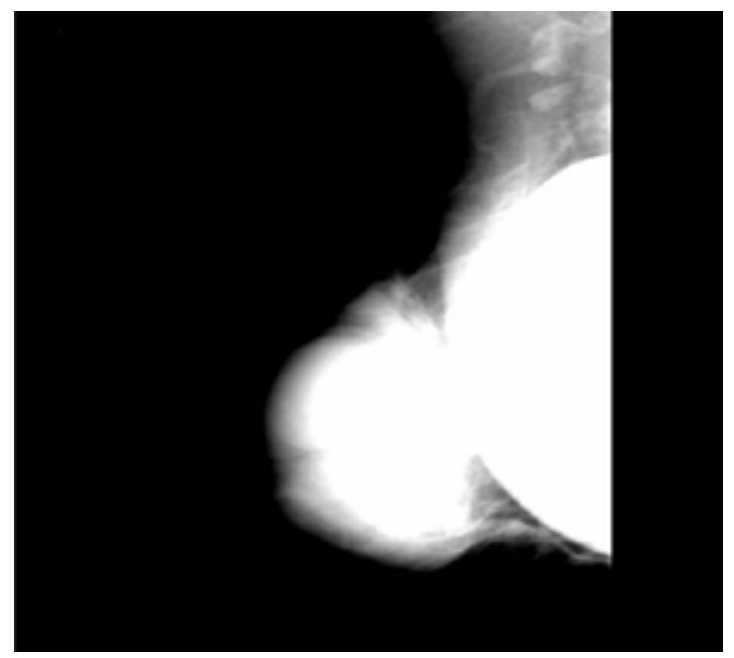

Figura 1. Mamografía: Se objetiva formación nodular de bordes bien delimitados que abarca prácticamente toda la mama.

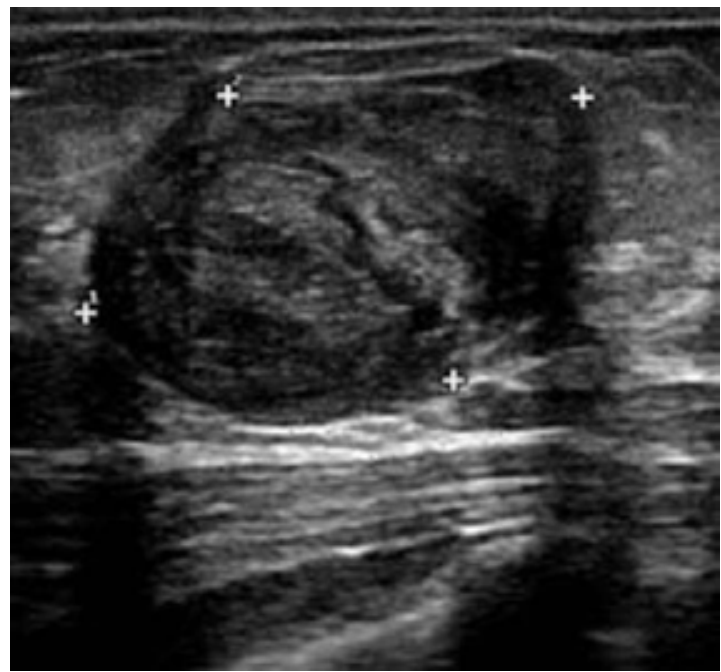

Figura 2. Ecotomografía mamaria: Formación nodular hipoecoica de bordes regulares indistinguible de fibroadenoma

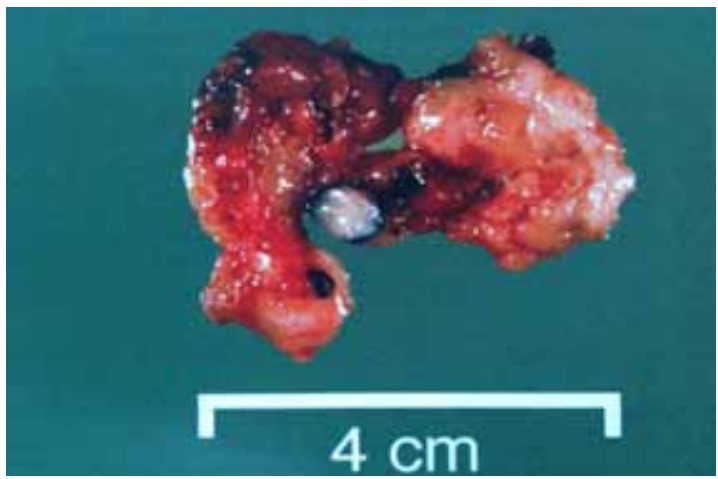

Figura 3. Lesión de aspecto lobulado indistinguible de un fibroadenoma.

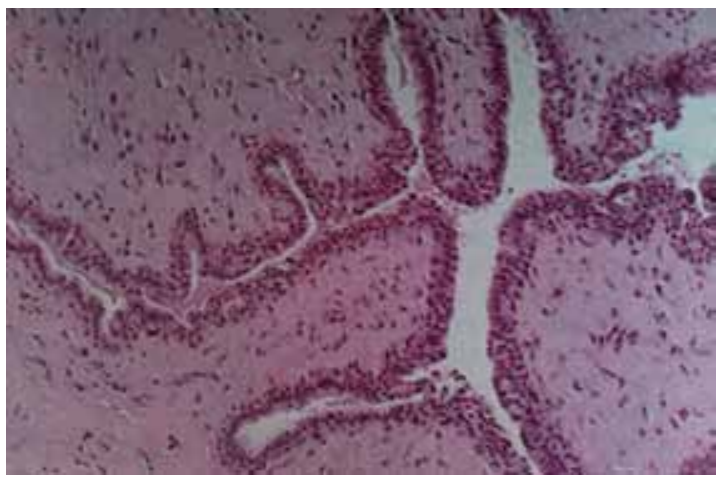

Figura 4. Proyecciones papilares de epitelio estromal intercalando zonas de hiperplasia y atipia. 
Las formas benignas se caracterizan por un incremento del epitelio estromal con un grado moderado de atipia celular, márgenes circunscritos y baja tasa de mitosis (menos de 4 mitosis por campo). En cambio, las formas malignas se caracterizan por marcado tejido estromal con atipia, infiltración de márgenes y alta tasa de mitosis (>de 10 mitosis por campo). Se ha sugerido, por tanto, que el sobrecrecimiento estromal se relaciona con la agresividad de la enfermedad y el desarrollo de enfermedad metastásica.

La determinación de ciertos marcadores tumorales tales como el $\mathrm{P} 53, \mathrm{KI}-67$, factor de crecimiento epitelial, entre otros, permiten predecir el comportamiento y pronóstico de la enfermedad. Se ha visto que la expresión del factor de crecimiento vascular endotelial indica la presencia de tumores Phyllodes de alto grado, siendo más reactivo en los estromas de los malignos que en los benignos. EI CD 10 también presenta reactividad en las células estromales de los tumores Phyllodes, siendo más frecuente en los malignos. Las formas malignas presentan metástasis por vía hematógena principalmente a pulmón y hueso, siendo raro la afectación ganglionar ( $<$ de $1 \%$ de los casos).

En cuanto al tratamiento, la escisión local de la lesión, tanto para formas benignas y malignas, con márgenes de al menos $1 \mathrm{~cm}$ es el tratamiento de elección, puesto que se ha visto que no existen diferencias estadísticamente significativas en aquellas pacientes tratadas con tratamiento conservador (con márgenes adecuados) frente a las pacientes tratadas con mastectomía. En aquellos casos en los que los márgenes de seguridad son $<1 \mathrm{~cm}$ se recomienda ampliación quirúrgica, dado que el principal factor determinante en las tasas de recurrencia es la existencia de márgenes incorrectos (14). Como ya se ha dicho, la afectación ganglionar es rara, por lo que no está indicada la realización de linfadenectomía.

El uso de radioterapia en esta entidad es controvertido. No está recomendado en las formas benignas, sin embargo, se ha mostrado eficaz para disminuir las tasas de recurrencia en las formas borderline y malignas (15). En lo que se está de acuerdo, es su aplicación en todos aquellos casos en los que es imposible obtener márgenes quirúrgicos adecuados, siendo más controvertido su uso en lesiones con márgenes adecuados. En nuestra serie la totalidad de las pacientes diagnosticadas de tumor Phyllodes maligno recibieron tratamiento radioterápico.

Se recomienda el uso de quimioterapia, tras valorar riesgos y beneficios, en pacientes con tumores malignos, pacientes con alto riesgo de recurrencia o lesiones de gran tamaño.

El pronóstico es excelente en las formas benignas, tal y como se comprueba en nuestra serie. Se obtienen tasas de curación del $100 \%$ en las formas benignas y las borderline tratadas de forma conservadora (con márgenes correctos); encontrando tasas de supervivencia libre de enfermedad a los 5 años en las formas malignas en torno al $60-80 \%$ $(2,16)$. Presenta una recurrencia local de un $15 \%$ y una recurrencia a distancia del 5 al $10 \%$.

\section{CONCLUSIÓN}

Tras ésta revisión retrospectiva se concluye que los tumores Phyllodes son más frecuentes en la cuarta década de la vida. Se presentan como nódulos de rápido crecimiento, siendo su localización más frecuente en los cuadrantes supero externos. El diagnóstico diferencial se realiza con fibroadenomas, y su diagnóstico definitivo es anatomopatológico, no existiendo hallazgos patognomónicos en las pruebas de imagen. El tratamiento de elección es conservador con márgenes de seguridad de al menos $1 \mathrm{~cm}$, dejando el uso de radioterapia y quimioterapia para las formas agresivas.

\section{BIBLIOGRAFÍA}

1. Calhoun K, Lawton TJ, Kim JM, Lehman CD, Anderson BO. Phyllodes Tumors; in Harris J, Lippman ME, Osborne CK, Morrow M. Diseases of the breast. Philadelphia, Lippincott Williams and Wilkins, 2010, pp 781.

2. Macdonald OK, Lee CM, Tward JD, Chappel CD, Gaffney DK. Malignant phyllodes tumor of the female breast: association of primary therapy with cause-specific survival from the Surveillance, Epidemiology, and End Results (SEER) program. Cancer 2006;107:2127-33.

3. Reinfuss M, Mitus J, Duda K, Stelmach A, Ryś J, Smolak K. The treatment and prognosis of patients with phyllodes tumor of the breast: An analysis of 170 cases. Cancer 1996;77:910-6.

4. Geisler DP, Boyle MJ, Malnar KF, McGee JM, Nolen MC, Fortner SM, Broughan TA. Phyllodes tumors of the breast: a review of 32 cases. Am Surg 2000;66:360-6.

5. Tavassoli FA, Devilee P. Pathology and genetics of tumors of the breast and female genital organs. In: World Health Organization Classification of Tumors, Lyons, IARC Press, 2003. p99.

6. Barrio AV, Clark BD, Goldberg JI, Hoque LW, Bernik SF, Flynn LW, Susnik B, Giri D, Polo K, Patil S, Van Zee KJ. Clinicopathologic features and long-term outcomes of 293 phyllodes tumors of the breast. Ann Surg Oncol 2007; 14:2961-70.

7. Karim RZ, Gerega SK, Yang YH, Spillane A, Carmalt $\mathrm{H}$, Scolyer RA, Lee CS. Phyllodes tumors of the breast: a clinicopathological analysis of 65 cases from a single institution. Breast 2009; 18:165-70. 
8. Birch JM, Alston RD, McNally RJ, Evans DG, Kelsey AM, Harris M, Eden OB, Varley JM. Relative frequency and morphology of cancers in carriers of germline TP53 mutations. Oncogene 2001; 20:4621-8.

9. National Comprehensive Cancer Network (NCCN) guidelines. Breast Cancer Guideline 2007. Hallado en: www.nccn.org

10. Lee $\mathrm{AH}$. Recent developments in the histological diagnosis of spindle cell carcinoma, fibromatosis and phyllodes tumor of the breast. Histopathology 2008; 52:45-57.

11. Dillon MF, Quinn CM, McDermott EW, O'Doherty A, O'Higgins N, Hill AD. Needle core biopsy in the diagnosis of phyllodes neoplasm. Surgery 2006; 140:779-84.

12. de Roos, WK, Kaye, P, Dent, DM. Factors leading to local recurrence or death after surgical resection of phyllodes tumors of the breast. Br J Surg 1999;86:396.

13. Parker SJ, Harries SA. Phyllodes tumors. Postgrad Med J 2001;77:428-35.

14. Barth RJ Jr, Wells WA, Mitchell SE, Cole BF. A prospective, multi-institutional study of adjuvant radiotherapy after resection of malignant phyllodes tumors. Ann Surg Oncol 2009; 16:2288-94.

15. Pezner RD, Schultheiss TE, Paz IB. Malignant Phyllodes Tumor of the breast: local control rates with surgery alone. Int J Radiat Oncol Biol Phys 2008;71:710-3.

16. Confavreux C, Lurkin A, Mitton N, Blondet R, Saba C, Ranchère $D$, Sunyach MP, Thiesse $P$, Biron $P$, Blay JY, Ray-Coquard I. . Sarcomas and malignant phyllodes tumors of the breast- A retrospective study. Eur $\mathrm{J}$ Cancer 2006; 42:2715-21. 